\title{
CHROMOSPHERIC DYNAMICS
}

\author{
ROBERT J. RUTTEN \\ Sterrekundig Instituut \\ Postbus 80000 , NL 3508 TA Utrecht, The Netherlands
}

\begin{abstract}
This brief review advertises a breakthrough in solar dynamics: the identification of chromospheric Ca II $\mathrm{K}_{2 V}$ "grains" as manifestations of acoustic shocks. The successful numerical reproduction of the intricate spectral characteristics of $\mathrm{K}_{2 V}$ grain formation by Carlsson \& Stein shows that the non-magnetic solar chromosphere is pervaded by outward traveling shocks to such extent that static modeling is no longer acceptable. Dynamical modeling should soon reconcile the long-standing $\mathrm{Ca}$ II/CO dichotomy. The chromospheric dynamics of the magnetized regions remains enigmatic.
\end{abstract}

\section{Chromospheric Oscillations}

Figure 1 displays large chromospheric disparity between internetwork and network oscillations. The two regimes require separate discussion.

The internetwork ("cell interior") areas in the Ca II panels show that the chromospheric "three-minute" oscillation extends to frequencies well above the cutoff frequency $f_{\text {AC }} \approx 5.5 \mathrm{mHz}$. From photosphere (bottom-left panel) to chromosphere, the power peak shifts up and widens to $f=3-10 \mathrm{mHz}$. Phase and coherence studies show that the chromospheric $3-10 \mathrm{mHz}$ oscillations are coupled to same-frequency oscillations in the underlying photosphere, with increasing upward propagation for increasing frequency (e.g., Lites et al., 1993). The waveforms show increasing non-linearity with height. Chromospheric $(k, \omega)$ diagrams show pseudo $p$-mode ridges above the cutoff frequency and a concentration of inter-ridge power near the cutoff frequency (cf. Steffens et al., 1995a).

For the network area, the CaII panels show power primarily at frequencies $f<4 \mathrm{mHz}$. Phase difference and coherence spectra in Lites et al. (1993) show that these slow network modulations are not coupled to motions in the underlying photosphere. They have been noted before; their nature remains 

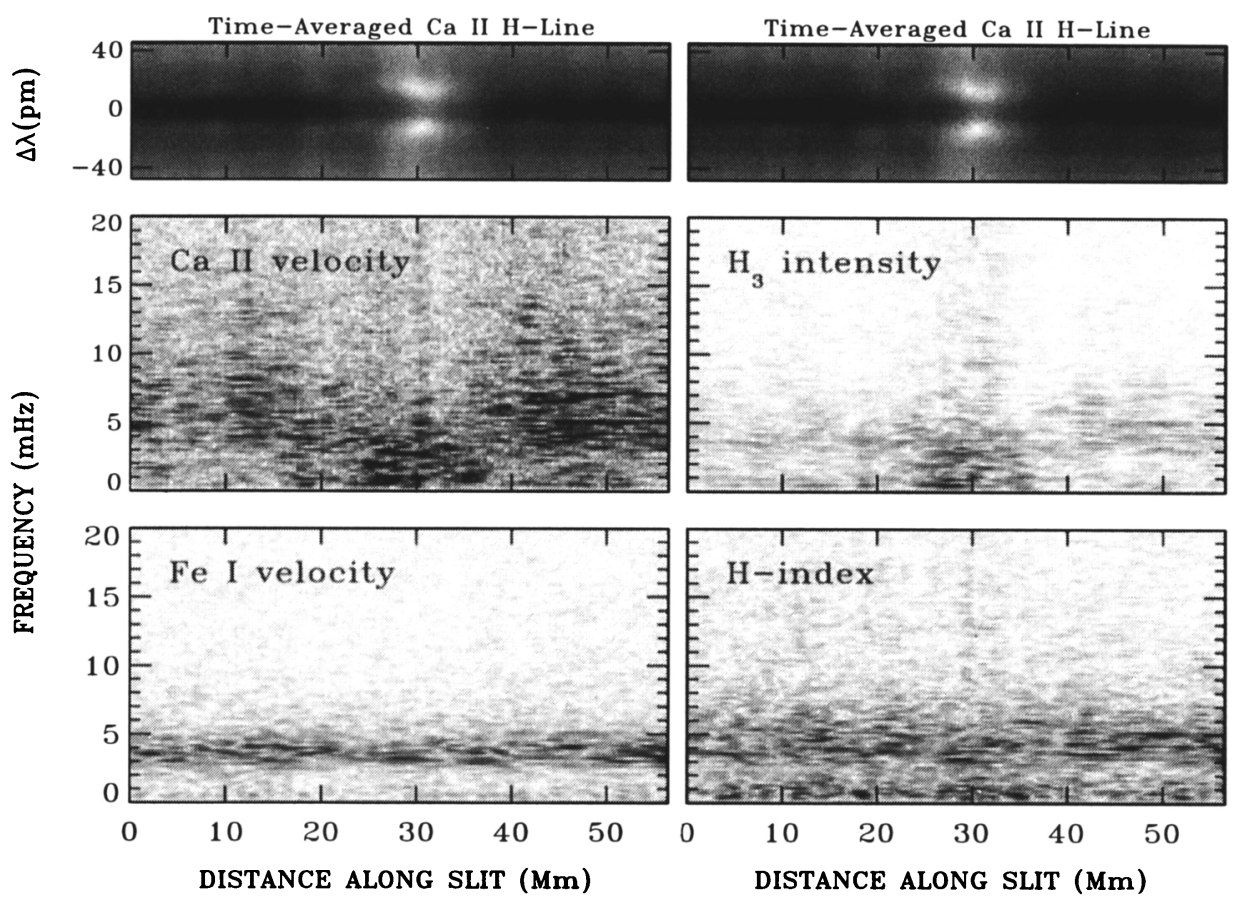

Figure 1. Chromospheric oscillations. The upper panels (identical, for easy reference) show time-averaged $\mathrm{Ca}$ II $\mathrm{H}$ line core profiles per spatial pixel along the spectrograph slit. The bright $\mathrm{H}_{2 V}$ and $\mathrm{H}_{2 R}$ emission around $x=30 \mathrm{Mm}$ marks a network area. The other panels are power spectra per pixel, with power coded as dark. The bottom left panel shows that the Dopplershift power for the photospheric FeI $396.682 \mathrm{~nm}$ line is confined to the five minute $(f=3.3 \mathrm{mHz})$ band of $p$-mode interferences. It does not show clear distinction between internetwork and network. The center panels for $\mathrm{Ca}$ II $\mathrm{H}_{3}$ Dopplershift (left) and intensity (right) show "three-minute" internetwork oscillations, especially in Dopplershift, and long-period modulations for the network. The bottom right panel shows $\mathrm{H}$-core intensity power across a $0.09 \mathrm{~nm}$ wide passband that is comparable to the transmission of the Mount Wilson H \& K photometer (cf. Fig. 2 of Rutten, 1994). This panel shows two bands of enhanced power without clear network/internetwork difference. The upper band consists of $p$-modes plus a sizable contribution from propagating acoustic waves. The lower band consists of gravity waves, prominent in the $\mathrm{H} \& \mathrm{~K}$ wings where these also show "reversed granulation". The apparent spatial coherence lengths of all these oscillation patterns are a few $\mathrm{Mm}(1 \mathrm{Mm} \approx 1.4 \operatorname{arcsec})$. From Lites et al. (1993).

unclear. Von Uexküll et al. (1989) attribute them to stochastic foot-point buffeting; Lites et al. (1993) find periodic behavior and invoke breaking gravity waves; Tsiropoula et al. (1994) display similar repetitiveness in $\mathrm{H} \alpha$ mottle flows.

The acoustic band in the bottom-right panel marks, presumably, the dynamical solar signature of the basal flux derived from cool-star activity relations (e.g., Schrijver et al., 1989). The gravity waves at the bottom have been seen before but not much studied. 

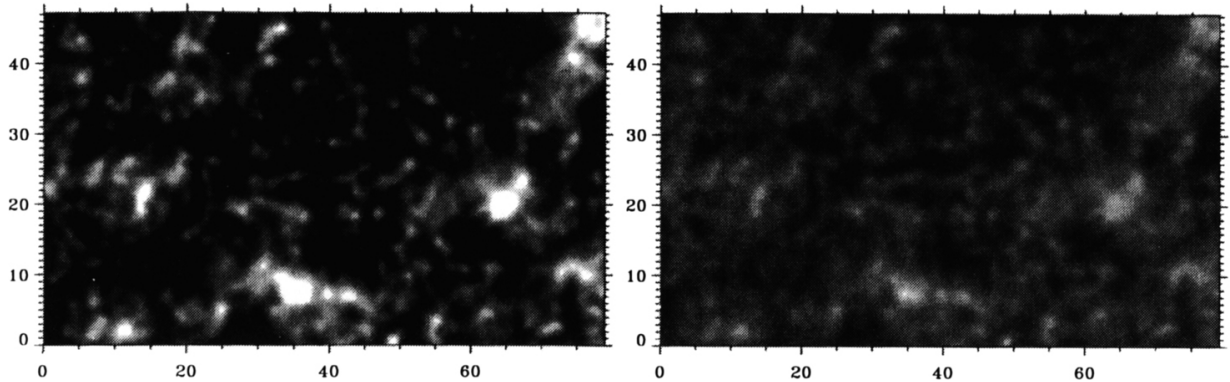

Figure 2. A tiny part of a famous Ca II $\mathrm{K}_{2 V}$ spectroheliogram taken by Gillespie at Kitt Peak twenty years ago. Larger parts have been published on the cover of Lites (1985), in Zirin (1988), in Rutten and Uitenbroek (1991b), as frontispiece to Solar Phys. 134, and elsewhere. Axis divisions in $\mathrm{Mm}$. The lefthand panel has enhanced contrast from clipping at bright and dark thresholds. Such high-gamma imaging shows $\mathrm{K}_{2 V}$ grains as minute bright points within the dark internetwork regions. On movies of such images the network appears as coarse-grained islands of stability, whereas the internetwork grains flash on and off in spidery, patchy patterns that often evolve rapidly, with apparent horizontal motions that can be highly supersonic. The righthand panel has not been thresholded and shows that all grains sit in larger patches of less enhanced brightness, marking them as "tips of the iceberg".

\section{Ca II $\mathbf{K}_{2 V}$ Grains}

The so-called CaII $\mathrm{H}_{2 V}$ and $\mathrm{K}_{2 V}$ grains provide rich diagnostics of the chromospheric oscillations in internetwork areas. The extensive older literature on this topic has been reviewed by Rutten and Uitenbroek (1991a). Much recent work, including the successful grain simulation of Carlsson and Stein (1994), is detailed in the highly recommended proceedings edited by Carlsson (1994) and reviewed less page-limitedly than here by Rutten (1995).

The lefthand panel of Fig. 2 shows why these chromospheric features have been described as distinctly identifiable entities since Hale's time. A few seem to possess a location memory and do in fact migrate as actual solar corks, following the mesogranular flow fields in the underlying photosphere (Brandt et al. 1992, 1994). The debate whether the other $\mathrm{K}_{2 V}$ grains mark sites of enhanced vertical field (Sivaraman and Livingston, 1982; Sivaraman, 1991) has not been definitively concluded, nor is the relationship with the small-scale internetwork patches of horizontal field recently discovered by Lites et al. (1996) clear.

The righthand panel of Fig. 2 shows that $\mathrm{K}_{2 V}$ grains are not isolated from their surroundings. Rutten and Uitenbroek (1991a) concluded that the grains mark pattern interference between the acoustic three-minute oscillations and $p$-mode oscillations, rather like waterspouts from wave interference on the ocean ("clapotis"). Since then, Kulaczewski (1992), von 

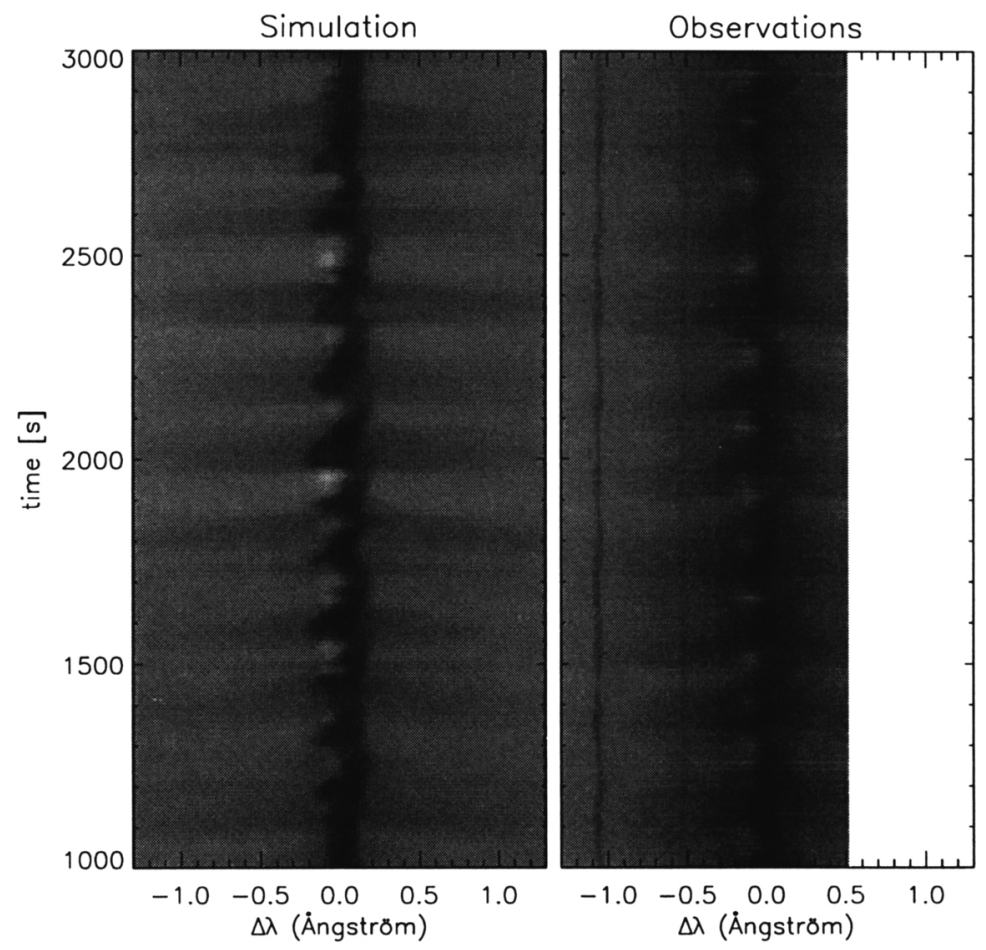

Figure 3. Ca II H profile evolution at one internetwork location, Right: data from Lites et al. (1993). Left: simulation results. From the cover of Carlsson (1994).

Uexküll and Kneer (1995), Steffens et al. (1995b) and Hofmann et al. (1995) have agreed with this suggestion on the basis of new observations. The numerical simulations of Carlsson and Stein (1994) prove it in detail.

\section{Carlsson-Stein Simulations}

The righthand panel of Fig. 3 shows the spectral evolution of $\mathrm{K}_{2 V}$ grains (in a format pioneered by Cram and Damé, 1983, and most beautifully displayed by Hofmann et al., 1995). It displays an intricate pattern of receding dark wing whiskers, sawtooth line-center shifts and bright $\mathrm{H}_{2 V}$ grain occurrences. Many efforts have been directed over the years towards reproducing this pattern from numerical modeling (cf. Rutten and Uitenbroek, 1991a). These have now culminated in the lefthand panel by Carlsson and Stein (1994). Their sub-photospheric piston was designed to reproduce the actually observed time-dependent Dopplershifts of the photospheric Fe I $396.682 \mathrm{~nm}$ line (Fig. 1). The broad-band piston excursions excite upwards propagating acoustic waves that steepen into weak shocks near 
$h=1000 \mathrm{~km}$. The shock temperatures are high because hydrogen ionization and recombination lag behind (Carlsson and Stein, 1992). NLTE-CRD modeling was then used to compute corresponding emergent $\mathrm{Ca} I \mathrm{II} \mathrm{H}$ profiles in the observational format of Fig. 3. The computed and observed grain evolution patterns are very similar; they agree fairly closely even in detail. Since these patterns - never adequately reproduced in previous modelingare so detailed, the good match establishes beyond doubt that $\mathrm{K}_{2 V}$ grains are made by acoustic shocks.

The informative Carlsson-Stein break-down diagram in Fig. 4 helps to understand the formation of a $\mathrm{H}_{2 V}$ grain in the computer. The diagram illustrates that the grains portray shocks at about $h=1 \mathrm{Mm}$. The distinctive blue-red asymmetry arises from the frequency-dependent vertical opacity scaling, set by the passages of previous shocks (upper-left panel). The notions of static plane-parallel line formation fail completely. Instead, the red side of the emergent line core "forms" over $\Delta h=700 \mathrm{~km}$; the $\Delta \nu=-11 \mathrm{~km} / \mathrm{s}$ downfall velocity at $h \approx 1.3 \mathrm{Mm}$ is not easily diagnosed from the emergent profile. On the blue side, the apparent Dopplershift of the $\mathrm{H}_{2 V}$ grain is skewed to $\Delta \nu=8 \mathrm{~km} / \mathrm{s}$ from the actual velocity $\Delta \nu=2 \mathrm{~km} / \mathrm{s}$ at the grain formation height.

Carlsson \& Stein's simulation is only one-dimensional, but their piston contains the local influences of ambient photospheric dynamics, including the local $p$-mode interference pattern, because it represents actual observations. The good agreement therefore implies that the chromospheric threeminute oscillation follows the underlying photosphere. A split-frequency pistoning experiment in Carlsson and Stein (1994) shows that the observed pattern is matched only by full-frequency pistons, with a dominating role by the photospheric three-minute components. A piston-repeat experiment shows that the pattern depends on the piston behavior only, not on the initial state of the chromosphere, if one allows for a 5-15 min delay that is needed by the piston to replace the initial state by its own shock sequences. Thus, chromospheric dynamics follows photospheric dynamics including $(k, \omega)$ patterning, but at some temporal delay and not in one-to-one frequency-by-frequency fashion.

Another informative Carlsson-Stein experiment (published also in Carlsson and Stein, 1995) consists of an emulation of the empirical VAL/FAL standard modeling by Avrett and co-workers (Fontenla et al., 1993), fitting time-independent temperature stratifications to the time-averaged ultraviolet continua computed from the time-dependent simulation. The Planck function in the ultraviolet is so non-linearly sensitive to temperature that the high shock temperatures require a chromospheric temperature rise in such spectrum fits, even though the actual time-averaged electron temperature does not rise significantly above the initial radiative-equilibrium model. 


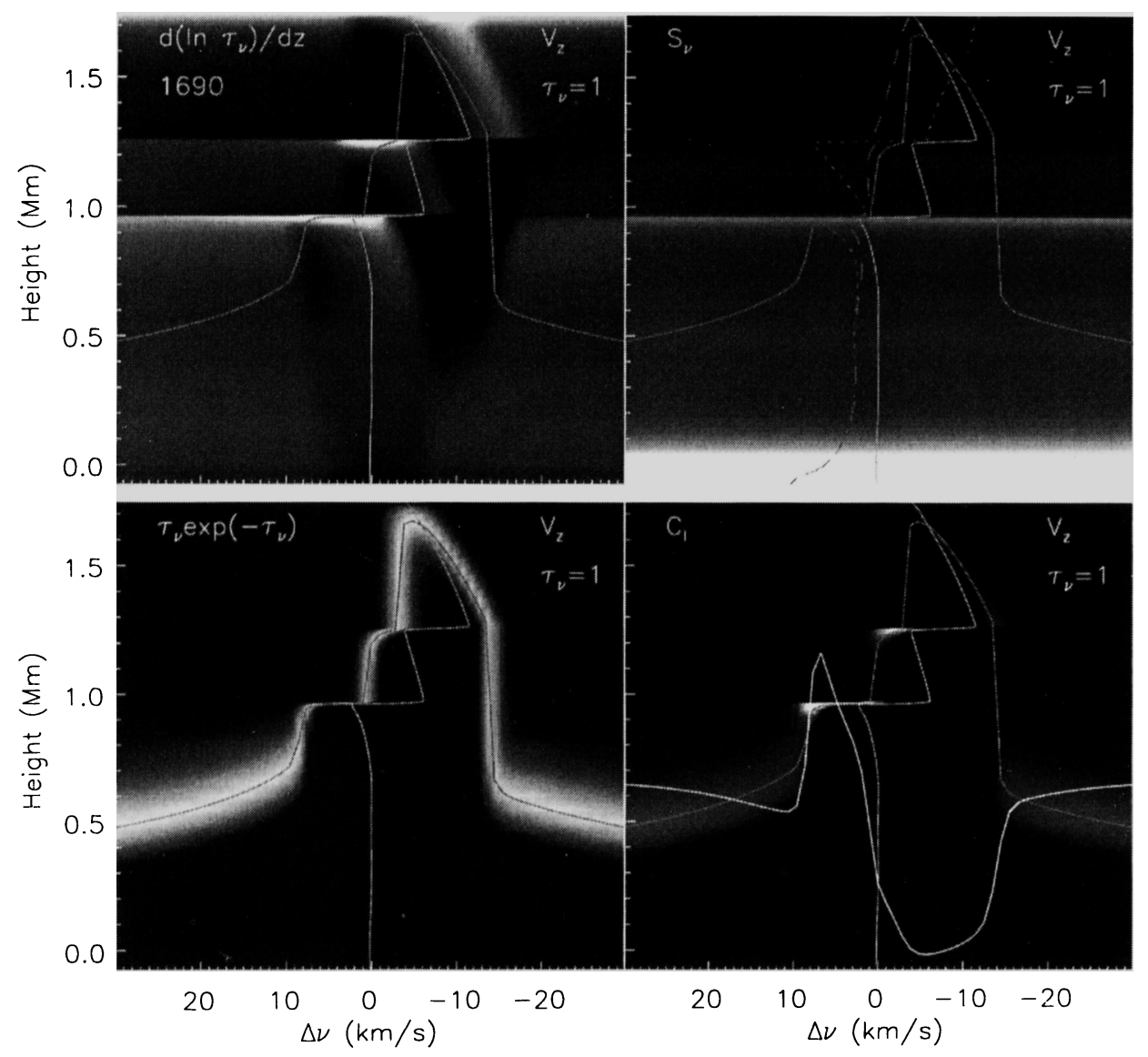

Figure 4. Ca II H line formation diagram for $t=1690 \mathrm{~s}$ in the simulation of Fig. 3. Bottom right panel: intensity contribution function $C_{I}$ (brightness coded), emergent profile (lower curve), vertical velocity $v_{z}$ (jagged curve, with two shocks), and $\tau_{\nu}=1$ height (thin curve through bright patches). The latter two curves are also shown in the other panels, which represent a breakdown of $C_{I}$ into the three brightness-coded factors

$C_{I}=\mathrm{d} / \mathrm{d} z \int j_{\nu} \exp \left(-\tau_{\nu}\right) \mathrm{d} z=\kappa_{\nu} S_{\nu} \exp \left(-\tau_{\nu}\right)=\left[S_{\nu}\right] \times\left[\tau_{\nu} \exp \left(-\tau_{\nu}\right)\right] \times\left[\mathrm{d} \ln \tau_{\nu} / \mathrm{d} z\right]$ with $j_{\nu}$ the emission coefficient, $\kappa_{\nu}$ the extinction coefficient, $S_{\nu}$ the total source function. The latter (upper right panel, brightness and dashed curve) drops from the Planck function (dotted) above $h=0.8 \mathrm{Mm}$ due to NLTE scattering, with considerable smoothing of the temperature spikes. Only the shock just below $h=1.0 \mathrm{Mm}$ leaves a marked $S_{\nu}$ increase (bright bar). It is constant across the panel because the simulation assumes complete redistribution over the line profile while the continuum contribution to $S_{\nu}$ is negligible at this height. The formation height factor $\tau_{\nu} \exp \left(-\tau_{\nu}\right)$ in the lower left panel peaks sharply around $\tau_{\nu}=1$. The steep drop at $\Delta \nu \approx-13 \mathrm{~km} / \mathrm{s}$ shows that this part of the profile "is formed" over $h=0.6-1.3 \mathrm{Mm}$ without height discrimination because the Doppler core shifts back abruptly near $h=1.3 \mathrm{Mm}$. In contrast, this factor peaks sharply at the grain-formation height $h=0.95 \mathrm{Mm}$ for $\Delta \nu \approx 8 \mathrm{~km} / \mathrm{s}$. The opacity scaling factor $\mathrm{d}\left(\ln \tau_{\nu}\right) / \mathrm{d} z=\kappa_{\nu} / \tau_{\nu}$ in the upper left panel is large at frequencies and heights with much extinction at small optical depth. It peaks in opaque layers with small overlying opacity. This factor causes considerable asymmetry. From Carlsson and Stein (1994). 
This experiment indicates that the long-lived "cool CO clouds" proposed by Ayres (1981) do not exist as spatially distinct features-as is indeed evident from Fig. 3 of Uitenbroek and Noyes (1994) - but that cool and hot material exist in temporal alternation along the line of sight. However, Ayres was right all along in that the non-magnetic low chromosphere is a rather cool place, without ubiquitous temperature rise. The problem lay in recognizing that the $\mathrm{H} \& \mathrm{~K}$ and ultraviolet diagnostics are nonlinearly sensitive to the shockingly noisy, clapotispheric nature of this "quiet" regime.

\section{Discussion}

The Carlsson-Stein simulation represents an exciting breakthrough, but is undoubtedly amenable to improvement in quantitative details. The computed time-averaged temperature is possibly too high due to the neglect of ultraviolet and infrared line cooling, the details of $\mathrm{Ca}$ II line formation-in particular oscillation phase differences between various Ca II lines (Skartlien et al., 1994)-depend on the questionable assumptions of complete redistribution and microturbulence, and the one-dimensional nature of the simulation inhibits reliable estimation of the energy balance. Nevertheless, the simulation shows how the Sun produces $\mathrm{K}_{2 V}$ grains, and so certifies that internetwork regions must be modeled time-dependently.

There are a number of interesting issues to study. This new view of the quiet chromosphere must be checked in detail against the other diagnostics of the upper photosphere and low chromosphere (the former temperature minimum region). Reconciliation with the $\mathrm{CO}$ data should come soon, with the latter probably adding accurate diagnostics of the time-averaged temperature (Avrett et al., 1995). The discordant phase behavior of the Na ID lines, which seems to indicate resonant oscillations in addition to propagating waves (Deubner et al., 1995), should be clarified. Higher up in the chromosphere, the formation of the $\mathrm{Mg}$ II $\mathrm{h} \& \mathrm{k}$ lines forms an obvious topic. The apparent connection, yet higher, between the three-minute oscillation and the $15-25 \mathrm{~km} / \mathrm{s}$ "chromospheric jets" observed in C I lines (Hoekzema, 1994) should be food for SOHO proposals.

More general questions are to wonder about the spatial distribution of piston properties over the solar surface (cf. Rutten, 1995), the role of these shocks in the energy budget of the quiet solar chromosphere, and, last but not least, how such shocks affect the chromospheres of other stars.

Acknowledgements. I thank Mats Carlsson and Bob Stein for permission to include their impressive results, Klaus Strassmeier for inviting me to give this review, Karel Schrijver for extensive comments, Bill Livingston for loan of Gillespie's spectroheliogram, Kevin Reardon for scanning it, and Karel Schrijver for measuring its scale. 


\section{References}

Avrett, E., Höflich, P., Uitenbroek, H., and Ulmschneider, P.: 1995, preprint

Ayres, T. R.: 1981, ApJ 244, 1064

Brandt, P. N., Rutten, R. J., Shine, R. A., and Trujillo Bueno, J.: 1992, in M. S. Giampapa and J. A. Bookbinder (Eds.), Cool Stars, Stellar Systems, and the Sun, Proc. Seventh Cambridge Workshop, Astron. Soc. Pac. Conf. Series 26, p. 161

Brandt, P. N., Rutten, R. J., Shine, R. A., and Trujillo Bueno, J.: 1994, in R. J. Rutten and C. J. Schrijver (Eds.), Solar Surface Magnetism, NATO ASI Series C 433, Kluwer, Dordrecht, p. 251

Carlsson, M. (Ed.): 1994, Chromospheric Dynamics, Proc. Mini-workshop, Inst. Theor. Astrophys., Oslo

Carlsson, M. and Stein, R. F.: 1992, ApJ 397, L59

Carlsson, M. and Stein, R. F.: 1994, in M. Carlsson (Ed.), Chromospheric Dynamics, Proc. Miniworkshop, Inst. Theor. Astrophys., Oslo, p. 47

Carlsson, M. and Stein, R. F.: 1995, ApJ 440, L29

Cram, L. E. and Damé, L.: 1983, $A p J$ 272, 355

Deubner, F.-L., Waldschik, T., and Steffens, S.: 1995, $A \& A$ in press

Fontenla, J. M., Avrett, E. H., and Loeser, R.: 1993, ApJ 406, 319

Hoekzema, N. M.: 1994, in M. Carlsson (Ed.), Chromospheric Dynamics, Proc. Miniworkshop, Inst. Theor. Astrophys., Oslo, p. 111

Hofmann, J., Steffens, S., and Deubner, F.-L.: 1995, $A \& A$ in press

Kulaczewski, J.: 1992, $A \& A$ 261, 602

Lites, B. W. (Ed.): 1985, Chromospheric Diagnostics and Modeling, Proceedings NSO Summer Conference, Sacramento Peak Observatory, Sunspot, New Mexico

Lites, B. W., Leka, K. D., Skumanich, A., Pillet, V. M., and Shimizu, T.: 1996, ApJ in press

Lites, B. W., Rutten, R. J., and Kalkofen, W.: 1993, ApJ 414, 345

Rutten, R. J.: 1994, in M. Carlsson (Ed.), Chromospheric Dynamics, Procs. Miniworkshop, Inst. Theor. Astrophys., Oslo, p. 25

Rutten, R. J.: 1995, in J. T. Hoeksema, V. Domingo, B. Fleck, and B. Battrick (Eds.), Helioseismology, Procs. Fourth SOHO Workshop, ESA SP-376 Vol. 1, ESA Publ. Div., ESTEC, Noordwijk, p. 151

Rutten, R. J. and Uitenbroek, H.: 1991a, Solar Phys. 134, 15

Rutten, R. J. and Uitenbroek, H.: 1991b, in P. Ulmschneider, E. R. Priest, and R. Rosner (Eds.), Mechanisms of Chromospheric and Coronal Heating, Proc. Heidelberg Conf., Springer Verlag, Berlin, p. 48

Schrijver, C. J., Dobson, A. K., and Radick, R. R.: 1989, ApJ 341, 1035

Sivaraman, K. R.: 1991, in P. Ulmschneider, E. Priest, and B. Rosner (Eds.), Mechanisms of Chromospheric and Coronal Heating, Heidelberg Conference, Springer Verlag, Berlin, p. 44

Sivaraman, K. R. and Livingston, W. C.: 1982, Solar Phys. 80, 227

Skartlien, R., Carlsson, M., and Stein, R. F.: 1994, in M. Carlsson (Ed.), Chromospheric Dynamics, Proc. Miniworkshop, Inst. Theor. Astrophys., Oslo, p. 79

Steffens, S., Deubner, F.-L., Hofmann, J., and Fleck, B.: 1995a, A\&A 302, 277

Steffens, S., Hofmann, J., and Deubner, F.-L.: 1995b, $A \& A$ in press

Tsiropoula, G., Alissandrakis, C. E., and Schmieder, B.: 1994, A\&A 290, 285

Uitenbroek, H. and Noyes, R. W.: 1994, in M. Carlsson (Ed.), Chromospheric Dynamics, Proc. Miniworkshop, Inst. Theor. Astrophys., Oslo, p. 129

von Uexküll, M. and Kneer, F.: 1995, $A \& A$ 294, 252

von Uexküll, M., Kneer, F., Malherbe, J. M., and Mein, P.: 1989, $A \& A$ 208, 290

Zirin, H.: 1988, Astrophysics of the Sun, Cambridge Univ. Press, U.K. 